\title{
Universidades y Desarrollo Económico Local El caso de dos centros de investigación de la Universidad de Concepción
}

\author{
Cristián Pincheira \\ Universidad de Concepción, Chile
}

\section{Resumen}

Esta investigación describe y compara los modelos de negocio de dos centros de investigación asociados a la Universidad de Concepción: la Unidad de Desarrollo Tecnológico y el Centro de Biotecnología. Se realizaron entrevistas semi estructuradas a una muestra de actores del Estado, Empresa y Universidad. Es posible afirmar que ambos centros de investigación, pese a tener propósitos similares y estar situados en un contexto común, son percibidos por sus actores clave como diferentes en su contribución al desarrollo económico local. La Unidad de Desarrollo Tecnológico es percibida como una iniciativa con mayor contribución al desarrollo económico local, principalmente por su enfoque hacia emprendedores y pequeñas y medianas empresas, además de ofrecer una propuesta de valor orientada a la oferta de soluciones a las necesidades y realidades locales, mientras que el Centro de Biotecnología se ha relacionado principalmente con la gran empresa, desarrollando investigación de excelencia. Se recomienda que iniciativas futuras estén orientadas al fortalecimiento y desarrollo de pequeña y mediana empresa.

Palabras clave: Desarrollo económico local, modelo Canvas, innovación.

\section{Universities and Local Economic Development. The Case of Two Research Centers associated to the University of Concepción}

\begin{abstract}
This research describes and compares the business models of two research centers associated to the University of Concepción: the Technological Development Unit and the Biotechnology Center. Semi structured interviews were made to a sample of actors from the State, Enterprise and University. We can say that both research centers, despite having similar purposes and be located in a common context, they are perceived by their stakeholders as different in their contribution to local economic development. The Technology Development Unit is perceived as an initiative with greater contribution to local economic development, mainly for its approach to entrepreneurs and small and medium enterprises, in addition to offering a value proposition focused on offering solutions to local needs and realities. On the other hand, the Biotechnology Center has been associated mainly with large companies, developing excellence research. It is recommended that future initiatives would be aimed to the strengthening and development of small and medium enterprises.
\end{abstract}

Keywords: Local economic development, Canvas model, innovation.

*Dirección de correspondencia [Correspondence address]: Cristián Pincheira, Universidad de Concepción, Chile

E-mail: cristpincheira@udec.cl 


\section{Universidad y desarrollo local}

La región de Biobío tiene un desarrollo industrial importante a nivel nacional y recursos naturales diversos desde el punto de vista de las ventajas comparativas y competitivas que aporta. La economía de la región está sustentada sobre la base de sectores económicos de gran desarrollo desde su aporte al PIB de la región, así como a la economía nacional (Industria manufacturera, Agricultura y Silvicultura, Energía). Consecuentemente, la economía regional está orientada al desarrollo de actividades económicas dirigidas a los mercados de exportación, donde participa activamente la producción forestal, pesquera e industrial.

Este desarrollo, no puede ser explicado sin hacer mención a la Universidad de Concepción, principal institución de educación superior solicitante de patentes a nivel nacional el año $2014^{1}$. Existe una intensa relación de sus académicos con el medio productivo y con comunidades regionales y nacionales, en particular en el ámbito de la innovación y el desarrollo social y económico, aspecto que se materializa en la interacción directa de Facultades y Centros de investigación con el medio para la ejecución de proyectos, la prestación de servicios, o bien por la participación de la Universidad en los programas para la creación de Centros Regionales, Consorcios Tecnológicos y Centros Basales. Dichas iniciativas constituyen vías a través de las cuales la Universidad, y la investigación que en ella se desarrolla, concretan su relación con el entorno, relación que ha sido vista como muy exitosa.

En este contexto, se observa que el vínculo entre desarrollo local y universidad, en la región de Biobío, es particularmente exitoso, y en este sentido necesario de estudiar. Este artículo caracteriza y describe el modelo de negocio de dos centros de investigación de carácter regional: la Unidad de Desarrollo Tecnológico (UDT) y el Centro de Biotecnología (CB), ambos dependientes de la Universidad de Concepción y enfocados en fortalecer la matriz productiva de la región, pero en los que se evidencian resultados dispares en relación a su vinculación e impacto en el fortalecimiento del sector productivo. De esta forma, a través de la comparación de ambos modelos de negocio, se busca identificar los elementos que diferencian y explican los distintos resultados obtenidos.

\footnotetext{
${ }^{1}$ http://www.udec.cl/panoramaweb2/2015/04/udecnuevamente-es-primera-en-patentes-a-nivel-pais/
}

\section{Desarrollo Económico Local}

En este artículo, se entiende por desarrollo económico local al proceso de crecimiento y cambio estructural que, mediante la utilización del potencial de desarrollo existente en el territorio, conduce a la mejora del bienestar de la población de una localidad o una región. Cuando la comunidad local es capaz de liderar el proceso de cambio estructural, la forma de desarrollo se puede convenir en denominarla desarrollo local endógeno.

La teoría económica neoclásica explica el crecimiento económico de un territorio por medio del Modelo de crecimiento de Solow (De Gregorio, 2007), el cual gira en torno a 4 variables: la producción, el stock de capital, el trabajo y un factor dependiente de la tecnología o eficiencia del trabajo. El modelo de Solow pretende explicar cómo crece la producción nacional de bienes y servicios mediante un modelo cuantitativo. En este modelo, el crecimiento económico se produce básicamente por la acumulación constante de capital. Si cada año aumenta la maquinaria y las instalaciones disponibles (capital fijo) para producir, se obtendrán producciones progresivamente mayores, cuyo efecto acumulado a largo plazo tendrá un notable aumento de la producción y, por tanto, un crecimiento económico notorio.

En desacuerdo al modelo de crecimiento económico neoclásico, se encuentra la teoría económica evolucionista (Palacios, 2005) que se opone a las ideas neoclásicas, como la racionalidad ilimitada, conocimiento omnisciente, optimización por parte de los individuos y homogeneidad de agentes. Estos modelos, plantean que el crecimiento económico tiene ciclos de comportamiento dinámicos, postulando la concepción de la empresa como el locus de la innovación tecnológica, así como su uso del concepto de destrucción creativa para describir los cambios producidos por estas innovaciones, donde los actores enfrentan alta incertidumbre sobre el resultado de sus acciones. Refuerza el rol de la innovación, la heterogeneidad a nivel país e industria, del conocimiento interactivo y de las empresas.

Este modelo de desarrollo económico, sirve como telón de fondo para nuevos modelos de innovación, los cuales nacen como crítica al llamado "modelo lineal de innovación" que separa investigación fundamental e investigación aplicada y la relación entre industria e investigación académica. Dentro de estos nuevos modelos de innovación, destaca el Modelo de la Triple Hélice. 


\section{Modelo de la Triple Hélice}

Este modelo, se centra en el análisis de las relaciones e interacciones mutuas entre las universidades y los entornos científicos, las empresas e industrias y los gobiernos. Atiende a las interacciones y comunicaciones entre actores e instituciones, pues asume que la innovación surge de las interacciones mutuas entre estas distintas esferas: el potencial para el conocimiento innovador, los recursos económicos y las posibilidades de mercado, y las normas e incentivos de las políticas públicas de innovación. La imagen de una triple hélice (inspirada en la biología), es una metáfora para expresar una alternativa dinámica al modelo de innovación imperante en las políticas de los años ochenta del siglo veinte, al tiempo que visualiza la complejidad inherente a los procesos de innovación (Etzkowitz et al., 000b).

Respecto a las relaciones que ocurren entre las distintas esferas, estas pueden tomar diversas formas, reflejando mecanismos sociales y económicos que inducen a cambiar el régimen de la triple hélice. La moderación de conflictos y colaboración es una forma específica de interacción tríadica de entidades, que tiene un mayor potencial de convertir la tensión y los conflictos de interés en la convergencia y confluencia alrededor de objetivos comunes y situaciones ganar-ganar.

La sustitución es otro tipo de interacción que se presenta cuando las esferas institucionales llenan vacíos de otras esferas, producto de la débil acción de estas últimas. Existe sustitución entre esferas, que es cuando una esfera asume actividades que normalmente le corresponden a otra, por ejemplo, las universidades, además de sus actividades de docencia e investigación, frecuentemente forman empresas y transfieren tecnologías, brindando apoyo e incluso financiamiento para propiciar emprendimientos, ejerciendo así un papel que tradicionalmente le corresponde a la industria. También está la sustitución dentro de las esferas, donde diferentes actores asumen actividades que tradicionalmente las realiza otro dentro de una misma esfera institucional, ejemplo de esta situación es cuando las instituciones de formación profesional toman la iniciativa por sobre las universidades de participar en iniciativas conjuntas con empresas locales.

Las redes de estructura formal e informal a nivel nacional, regional e internacional son otra importante forma de interacción entre los componentes del sistema de la triple hélice. Su agregación puede ser más fácil o difícil de identificar, dependiendo de la madurez de la red, su alcance, actividades y visibilidad en el dominio público. Si bien las redes no son un fenómeno especifico de las interacciones de la triple hélice, sí se encuentran frecuentemente en este tipo de interacciones.

Por otro lado, las funciones de los sistemas de la triple hélice se definen como un conjunto de procesos específicos que ocurren en los espacios de conocimiento, innovación y consenso.

El espacio del conocimiento es el conjunto de las actividades de generación, difusión y uso de conocimiento de los componentes. La construcción de este espacio es un paso esencial en la transición a una sociedad del conocimiento y tiene como último propósito crear una masa crítica de recursos para fortalecer la base de conocimiento local, regional y nacional, evitar la fragmentación y reducir la duplicación de esfuerzos de investigación.

El espacio de la innovación consiste en las actividades realizadas especialmente por las organizaciones multiesfera (híbridas), emprendedores individuales e instituciones, teniendo como finalidad la creación y desarrollo del potencial intelectual y empresarial, atracción de talento y empresas innovadoras de otros lugares, construyendo así una ventaja competitiva para la región y el país.

El espacio del consenso es el conjunto de actividades que reúnen a los componentes del sistema Triple hélice en una lluvia de ideas, discusión y evaluación de propuestas para el avance hacia un régimen basado en el conocimiento. Aun cuando la iniciativa proviene de un filamento particular de la Triple hélice, se necesita la participación de actores de otras esferas en un proceso de colaboración. A través de cross-fertilizing, diversas perspectivas pueden generar ideas y resultados que probablemente los actores no podrían haber generado individualmente.

Además de los componentes, relaciones y funciones, existen cuatro procesos relacionados con los principales cambios en la producción, intercambio y uso del conocimiento que ha identificado el modelo triple hélice. El primero es una transformación interna en cada una de las hélices, tales como el desarrollo de los lazos laterales entre empresas a través de alianzas estratégicas o una asunción de una misión de desarrollo económico por las universidades. El segundo es la influencia de una esfera institucional sobre otra, generando en la segunda una transformación. El tercero es la creación de un nuevo recubrimiento de vínculos trilaterales, redes y organizaciones entre las tres hélices, que sirven para institucionalizar y reproducir interfaz, así como estimular la creatividad organizativa y cohesión regional. Un cuarto proceso es el efecto recurrente de estas redes interinstitucionales del mundo académico, industrial y gubernamental tanto en su origen como esferas autónomas, como en la sociedad en general. 
Esto ha dado lugar a un cambio en el paradigma de cómo se concibe la Universidad actualmente. Etzkowitz y Leydesdorff (000a) plantea el modelo de "Universidad emprendedora", como un nuevo modelo de universidad y comportamiento interno de estas instituciones.

La Universidad Emprendedora incluye los siguientes mecanismos emergentes y de desarrollo de estructuras que se relacionan con los cuatro procesos señalados anteriormente:

- Transformación interna: Las tareas académicas tradicionales son redefinidas y ampliadas, de acuerdo a los requerimientos de las nuevas funciones.

- Interfaz de procesos: La Universidad Emprendedora requiere una mayor capacidad de inteligencia, vigilancia y negociación con otras esferas institucionales, especialmente la industria y el gobierno. Más allá de la capacidad de la dirección superior de la Universidad a comprometerse con sus contrapartes en otras esferas institucionales, una capacidad de vinculación organizacional nivel medio da a la Universidad la capacidad de identificar la confluencia de intereses entre los académicos y organizaciones externas contrapartes.

- Efectos recursivos: Más allá de establecer vínculos con las organizaciones existentes, la Universidad como empresario también desarrolla capacidades para ayudar a la creación de nuevas organizaciones. Esto puede tomar varias formas tales como formación de las empresas basadas en investigación académica y liderazgo en la formación de organizaciones regionales, trayendo el diversos ámbitos institucionales por lo común propósito de fomentar la innovación.

Esta transformación en los propósitos, funciones y relaciones, han dado paso a un cambio de paradigma en la concepción de las Universidades, donde además de las dos misiones históricas, entregar enseñanza y producir conocimientos, es necesario añadir otra misión que considere las actividades de extensión que buscan lograr un impacto en su contexto económico regional. El objetivo en esta tercera misión es crear una nueva estrategia de la universidad que esté más cerca de los problemas de la sociedad y dirigirla hacia una contribución más práctica en su región.

\section{Metodología}

Se utilizó una metodología cualitativa, con el objetivo de describir resultados exploratorios. Es decir, esta investigación no tiene una pretensión generalizadora, y más bien busca comprender, interpretar, describir y explicar las características que diferencian a dos centros con objetivos similares, pero con resultados diferentes, encontrándose dentro de la misma área económica de la Región de Bío-bío.

Como técnica de recolección de información, se aplicó una entrevista semiestructurada a una muestra de entrevistados seleccionados mediante un muestreo por conveniencia. En estas entrevistas se levantó la información necesaria para construir los modelos de negocio de cada uno de los centros, así como elementos relevantes de los vínculos entre una universidad regional y fortalecimiento del sector productivo local, a considerar en la generación de políticas públicas en esta materia. La siguiente tabla resume el número de entrevistas que se realizaron.

La elección de dos centros de investigación, UDT y CB se basa en los siguientes argumentos:

- La principal actividad económica de la región del Biobío, dejando fuera el sector de servicio, es el área forestal y la manufactura con materia prima de origen forestal, abarcando en su conjunto el $47 \%$ del PIB regional (Banco Central de Chile, 2013)

- La Universidad de Concepción cuenta con 13 centros de $\mathrm{I}+\mathrm{D}+\mathrm{i}$, dos de los cuales trabajan en la investigación y desarrollo de nuevos procesos y productos asociados a la biomasa forestal como principal materia prima. Estos centros son el Centro de Biotecnología (CB) y la Unidad de Desarrollo Tecnológico (UDT).

- La principal fuente de financiamiento de ambos centros se basa en la adjudicación de proyectos de investigación concursables, provenientes de las agencias públicas de fomento a la investigación, desarrollo e innovación.

- Muchas de las empresas forestales y manufactureras presentes en la región han trabajado desarrollando proyectos con ambos centros, destacando las principales empresas del rubro: Masisa, CMPC y Arauco.

La siguiente tabla describe la visión y misión de cada centro: 
Tabla 1: Número de entrevistados

\begin{tabular}{|l|c|}
\hline Categoría & Número de entrevitados \\
\hline Investigador Centro A & 7 \\
\hline Investigador Centro B & 6 \\
\hline Funcionario Público & 6 \\
\hline Empresarios & 6 \\
\hline Total & $\mathbf{2 5}$ \\
\hline
\end{tabular}

Fuente: Elaboración propia

Tabla 2: Visión y Misión Centros de Investigación analizados.

\begin{tabular}{|c|c|c|}
\hline & Unidad de Desarrollo Tecnológico & Centro de Biotecnología \\
\hline Visión & $\begin{array}{l}\text { "Ser el centro científico, tecnológico y } \\
\text { de innovación líder en Chile y con re- } \\
\text { conocimiento internacional, en ámbitos } \\
\text { relacionados con la conversión de bio- } \\
\text { masa en productos de interés y valor } \\
\text { comercial" }\end{array}$ & $\begin{array}{l}\text { "Ser una institución líder en investigación, forma- } \\
\text { ción, transferencia e innovación en biotecnología de } \\
\text { recursos naturales renovables, para contribuir de ma- } \\
\text { nera eficiente e innovadora, al desarrollo económico } \\
\text { y social del país" }\end{array}$ \\
\hline Misión & $\begin{array}{l}\text { "Contribuir al desarrollo de Chile me- } \\
\text { diante la creación de conocimiento } \\
\text { científico e innovaciones tecnológicas en } \\
\text { el ámbito de las biorrefinerías forestales, } \\
\text { promoviendo su uso en empresas exis- } \\
\text { tentes y/o a través de nuevos empren- } \\
\text { dimientos" }\end{array}$ & $\begin{array}{l}\text { - Desarrollar investigación biotecnológica de excelen- } \\
\text { cia. } \\
\text { - Contribuir a la formación de capital humano es- } \\
\text { pecializado en la aplicación de herramientas biotec- } \\
\text { nológicas. } \\
\text { - Realizar transferencia tecnológica al sector produc- } \\
\text { tivo. } \\
\text { - Generar redes de colaboración, a través de la in- } \\
\text { teracción con grupos de investigación, nacionales e } \\
\text { internacionales. } \\
\text { - Promover y participar en el desarrollo y utilización } \\
\text { de la Biotecnología como herramienta para el desa- } \\
\text { rrollo económico y social del país. }\end{array}$ \\
\hline
\end{tabular}

Fuente: Elaboración propia

\section{Metodología de Canvas para la cons- trucción de modelos de negocio}

Un modelo de negocio es un conjunto de suposiciones acerca de cómo se desempeñará una organización, creando valor para todos los agentes de que depende, no solo para sus clientes. Osterwalder y Pigneur (2011) proponen una metodología basada en nueve elementos esenciales e interrelacionados presentes en cualquier organización, los cuales los expone en un lienzo (Canvas) para su análisis. Estos nueve elementos, son los siguientes:

- Segmentos de mercado

- Propuestas de valor

- Canales

- Relaciones con clientes

- Fuentes de ingresos

- Recursos clave

- Actividades clave
- Asociaciones clave

- Estructura de costos

A partir de estos nueve elementos, se compararán los modelos de negocio de los dos casos escogidos, buscando identificar los elementos que diferencian y explican los distintos resultados obtenidos.

\section{Resultados}

La siguiente tabla resume los modelos de negocio de cada uno de los centros de investigación:

\section{Clientes}

Si bien ambos centros trabajan con empresas similares (empresas forestales, biomateriales, biocombustibles, etc.), la UDT ha enfatizado su trabajo con pequeñas y medianas (PYME's) empresas asociadas al uso de la biomasa forestal, agilizando 
Tabla 3: Modelos de Negocio.

\begin{tabular}{|c|c|c|}
\hline & Unidad de Desarrollo Tecnológico & Centro de Biotecnología \\
\hline $\begin{array}{l}\text { Segmento de } \\
\text { clientes }\end{array}$ & $\begin{array}{l}\text { - Medianas y pequeñas (biomasa forestal) } \\
\text { - Emprendedores }\end{array}$ & $\begin{array}{l}\text { - Grandes empresas forestales } \\
\text { - Estudiantes de pre y post grado }\end{array}$ \\
\hline $\begin{array}{l}\text { Propuestas } \\
\text { de valor }\end{array}$ & $\begin{array}{l}\text { - Ofrecer soluciones a las necesidades de las em- } \\
\text { presas relacionadas con la biomasa forestal me- } \\
\text { diante el desarrollo de proyectos de investigación } \\
\text { y desarrollo tecnológico, mejorando sus procesos } \\
\text { y/o desarrollando nuevas líneas de productos } \\
\text { - Asistir técnicamente a emprendedores para el } \\
\text { desarrollo de sus ideas de negocio }\end{array}$ & $\begin{array}{l}\text { - Desarrollo de investigación biotecnológica de ex- } \\
\text { celencia para el mejoramiento del uso de la mate- } \\
\text { ria prima, agregando valor a la producción actual } \\
\text { y/o generando nuevos productos. } \\
\text { - Ofrecer la infraestructura, equipamiento y capi- } \\
\text { tal humano para el desarrollo de proyectos de te- } \\
\text { sis, en un ambiente académico de excelencia fuer- } \\
\text { temente vinculado con el sector productivo }\end{array}$ \\
\hline Canales & $\begin{array}{l}\text { - Canales tradicionales (visitas, reuniones, correos } \\
\text { electrónicos, página web) } \\
\text { - Concursos } \\
\text { - Contactos centralizados en dirección }\end{array}$ & $\begin{array}{l}\text { - Contactos particulares de cada investigador } \\
\text { - Canales tradicionales (visitas, reuniones. correos } \\
\text { electrónicos, página web) } \\
\text { - Newsletter } \\
\text { - Cafés científicos }\end{array}$ \\
\hline $\begin{array}{l}\text { Relación con } \\
\text { los clientes }\end{array}$ & $\begin{array}{l}\text { - Con la empresa medianas y pequeña transferi- } \\
\text { mos y asistimos, ellas no tienen capacidades de } \\
\text { investigación }\end{array}$ & $\begin{array}{l}\text { - Con las empresas forestales grandes hay co- } \\
\text { creacion }\end{array}$ \\
\hline $\begin{array}{l}\text { Fuentes de } \\
\text { ingreso }\end{array}$ & $\begin{array}{l}\text { - } 67 \% \text { aporte estatal (fondo basal y proyectos } \\
\text { Corfo) } \\
\text { - } 33 \% \text { aporte privado (empresas) ya sea por exi- } \\
\text { gencia de un proyecto, por solicitud de investiga- } \\
\text { ción de las empresas o por asistencia técnica }\end{array}$ & $\begin{array}{l}\text { - } 70 \% \text { aprox. Ingreso proveniente de proyectos } \\
\text { con financiamiento estatal (Fondecyt y Fondef) } \\
\text { - } 30 \% \text { aprox. Aporte empresas privadas y Univer- } \\
\text { sidad de Concepción }\end{array}$ \\
\hline $\begin{array}{l}\text { Recursos cla- } \\
\text { ves }\end{array}$ & $\begin{array}{l}\text { - Investigadores e ingenieros con dedicación ex- } \\
\text { clusiva UDT } \\
\text { - Capacidad (infraestructura) de escalamiento } \\
\text { - Unidad de Transferencia Tecnológica } \\
\text { - Liderazgo del director }\end{array}$ & $\begin{array}{l}\text { - Académicos UdeC } \\
\text { - Infraestructura y equipamiento de primer nivel } \\
\text { - Prestigio UdeC }\end{array}$ \\
\hline $\begin{array}{l}\text { Actividades } \\
\text { claves }\end{array}$ & $\begin{array}{l}\text { - Identificación de necesidades del sector produc- } \\
\text { tivo, con fuerte relación con la empresa } \\
\text { - Patentamiento y transferencia tecnológica } \\
\text { - Formulación y adjudicación de proyectos Corfo }\end{array}$ & $\begin{array}{l}\text { - Generación de ideas y presentación de propuesta } \\
\text { a las empresas } \\
\text { - Producción de artículos científicos } \\
\text { - Formulación y adjudicación de proyectos con } \\
\text { fondos Conicyt }\end{array}$ \\
\hline $\begin{array}{l}\text { Aliados cla- } \\
\text { ves }\end{array}$ & $\begin{array}{l}\text { - Agencias de gobierno relacionadas con el desa- } \\
\text { rrollo de ciencia y tecnologías } \\
\text { - Académicos Facultad de Ingeniería UdeC } \\
\text { - Los institutos de la organización alemana Fraun- } \\
\text { hofer Gesellschaft }\end{array}$ & $\begin{array}{l}\text { - Agencias de gobierno relacionadas con el desa- } \\
\text { rrollo de ciencia y tecnologías } \\
\text { - Facultad de Ciencias Forestales UdeC } \\
\text { - Facultad de Ciencias Naturales y Oceano- } \\
\text { grafícas UdeC }\end{array}$ \\
\hline $\begin{array}{l}\text { Estructura } \\
\text { de costos }\end{array}$ & - $60 \%$ de los costos son remuneraciones & $\begin{array}{l}-50 \% \text { de los costos son remuneraciones } \\
-50 \% \text { de los costos son equipamiento e insumos }\end{array}$ \\
\hline
\end{tabular}


el trabajo e incrementando así la cubertura de su trabajo en el sector productivo local. Por otro lado, el CB no acostumbra a trabajar en proyectos pequeños en el área forestal, sino que en la mayoría de los casos se ha involucrado en proyectos más competitivos, como los consorcios tecnológicos, donde participan principalmente empresas de gran tamaño, con capacidades de investigación y absorción de tecnologías. Son apuestas a largo plazo, lo que implica mayor complejidad y burocracia.

Por otro lado, dentro del cumplimiento de la tercera misión de las universidades, se encuentra la generación de empresas Spin-Off y Start-Up, labor que la UDT desarrolla al enfocar parte de sus esfuerzos a emprendedores internos y externos, ofreciendo, entre otras cosas, las capacidades de pilotaje y escalamiento. Por otro lado, la vinculación del CB con emprendedores, ha sido marginal pese a encontrarse inserto dentro del Campus de la Universidad de Concepción. La inserción en un ambiente académico, más que atraer a emprendedores, los aleja al no existir un canal institucional para generar el vínculo.

"La universidad carece de una ventanilla única donde pueda acudir a solicitar ayuda, para ir a la universidad deben golpear muchas puertas, por eso vienen para acá".

(E.R., Gerente de Investigación y desarrollo, Industria)

Finalmente, un tercer cliente que está siendo abordado con mayor énfasis por el CB que por la UDT, son los estudiantes de pre y postgrado. La cercanía geográfica y el vínculo de los investigadores del CB con la Universidad de Concepción, ha facilitado que este centro lleve a acabo de mejor manera la formación de capital humano, destacándolos como unos de sus clientes relevantes. No ocurre los mismo con la UDT cuando piensan en sus clientes, si bien existen proyectos de tesis de pre y postgrado realizados en la UDT, su ubicación geográfica e independencia han mermado la vinculación académica del centro con la universidad, situación que se vio atenuada a partir del proyecto basal adjudicado, generando vínculos principalmente con la facultad de Ingeniería de la Universidad de Concepción, pero aún no relevados en las entrevistas.

\section{Propuesta de valor}

La UDT se ha caracterizado, más que por su investigación, por el desarrollo tecnológico para la industria relacionada con la biomasa forestal, abordando este desarrollo en 4 líneas diferentes, pero todas en relación a una materia prima específica, abordando la investigación desde un enfoque aplicado. En cambio, al CB le ha faltado claridad en cuanto a su propósito. Dado que su creación involucró grandes inversiones del gobierno regional, este centro se proyectó como el centro de $\mathrm{I}+\mathrm{D}$ para el desarrollo regional, por lo que sus áreas de investigación abarcaron diversos sectores productivos de la región, diluyendo así su propósito. Sin embargo, la investigación básica y la producción de artículos científicos es un punto donde el CB destaca por sobre la UDT.

Se podría decir que el CB se ha convertido en una especie de asesor científico de la industria forestal, creando nuevo conocimiento, generando alianzas con grandes empresas de la región, desarrollando investigación, pero con bajo nivel de aplicabilidad; mientras que la UDT es vista por las empresas de la región como la unidad de desarrollo de nuevas tecnologías, donde empresas de todo tamaño van a escalar sus propias ideas y/o ideas desarrolladas en conjunto con el centro, bajo condiciones similares a las que presenta la realidad, convirtiéndose en la unidad de investigación que la mayoría de las empresas carecen.

En materia de emprendimiento, la UDT tiene un marcado enfoque en la creación de nuevas empresas internas y asesoramiento para la formación de nuevos emprendimientos externos, para lo cual ofrece su capacidad de escalamiento y pilotaje dada la infraestructura del centro y expertiz de sus investigadores y profesionales. En cambio, el foco del CB ha estado puesto en la investigación y vinculación con grandes empresas, descuidando la formación y asesoramiento a empresas más pequeñas.

Finalmente, el fuerte vínculo que tiene con la Universidad de Concepción, producto de la dependencia financiera y espacial, ha permitido al CB entregar una propuesta de valor a los estudiantes de pre y postgrado mucho más robusta y clara que la UDT, formando capital humano avanzado para la industria forestal regional y nacional.

\section{Canales}

Los canales utilizados en ambos centros son bastantes similares, las redes se forman a través de los investigadores y profesionales que trabajan en ellos sin estar necesariamente institucionalizadas, sin embargo el estilo gerencial de la dirección de la UDT de cierta manera centraliza las redes de contacto del centro.

Por otro lado, el CB ha desempeñado una mejor labor en la difusión de su quehacer, por medio del 
Newsletter y los cafés científicos que desarrolla durante el año, en el cual se convoca a la comunidad en general y se realiza un acercamiento a la labor del centro como a la ciencia en general.

Finalmente, la ubicación geográfica es determinante en la utilización de los canales de comunicación con sus clientes. La UDT se ubica en el parque industrial de Coronel, donde se ubican también un número importante de empresas de la región relacionadas con la industria forestal, lo que facilita el contacto con la industria, mientras que el CB se encuentra emplazado en el campus de la Universidad de Concepción, dentro de un entorno netamente académico, lo que muchas veces produce rechazo al sector empresarial, dificultando el contacto con ellos.

\section{Relación con los clientes}

La relación con los clientes viene determinada por el tipo de cliente en que pone énfasis cada centro. En el caso de la UDT, si bien trabaja con empresas grandes, medianas y pequeñas, su énfasis se centra en el trabajo y apoyo a las medianas y pequeñas empresas, transformándose como una especie de unidad de desarrollo tecnológico de dichas empresas. Con ellas se genera una relación de colaboración y transferencia tecnológica formal, la que ha ido forjando con el tiempo una relación de confianza y fidelización con gran parte de ellas. El enfoque empresarial de la gestión de la UDT, ha facilitado el vínculo universidad-empresa, intentando acomodar de mejor manera las dinámicas de trabajo y los tiempos en que se maneja la industria.

El CB por su parte, ha enfatizado en trabajar con las grandes empresas forestales de la región y el país, transformándose en una especie de asesor científico de éstas. Dado que las empresas de mayor tamaño poseen las capacidades necesarias para co-crear y absorber el trabajo desarrollado por el centro, la transferencia se realiza en su mayoría de manera informal, al formar universidad y empresas partes de un mismo equipo de trabajo. Si bien se han generado confianzas entre la gran industria forestal y el $\mathrm{CB}$, el marcado enfoque académico de este último, ha entorpecido el trabajo y logro de resultados oportunos con las empresas, generando a veces resistencia a trabajar en futuros proyectos.

\section{Fuentes de ingreso}

En relación a las fuentes de ingreso de cada centro, existen grandes diferencias que han marcado el quehacer. En primer lugar, la UDT el año 2007 se adjudicó un financiamiento basal de aproximadamente $\$ 4.000 .000 .000$ por un periodo de 5 años. Esto, le ha permitido cubrir sus gastos operacionales, aumentar y mejorar su planta de investigadores y profesionales y entregarle sustentabilidad a sus proyectos, presentado en un plan estratégico, junto con la autogestión financiera que este centro tiene desde sus inicios, lo que lo ha dotado de autonomía y capacidades para identificar y captar los recursos necesarios para su funcionamiento. En adición a esto último, UDT financia gran parte de sus proyectos con instrumentos Corfo, lo que de por sí determina capacidades especificas en los centros de investigación, como la vinculación con la empresas y la transferencia tecnológica.

Por otro lado, el CB desde su inicio ha sido financiado en gran parte por la Universidad de Concepción, ya sea mediante el "financiamiento basal" que esta entrega para gastos operacionales y administrativos, como por medio de las facultades que pagan los salarios de la mayoría de los investigadores, adscritos a alguna facultad. Esto ha significado al CB depender y alinearse con los propósitos de múltiples actores, restringiendo sus capacidades y autonomía necesaria para plantearse como un centro capaz de impactar en el sector productivo regional y nacional. Además, a diferencia de la UDT, el $\mathrm{CB}$ ha utilizado como instrumentos principales de financiamiento de proyectos los ofrecidos por Conicyt, como Fondef o Fondecyt, los que han fortalecido sus capacidades investigativas, pero a su vez han perjudicado las capacidades del CB de transferir su trabajo al sector productivo.

"[El Centro de Biotecnología] solo se abastece de Fondef, no sabe trabajar con los instrumentos de Corfo."

$$
\text { (J.C., Directivo, UDT y CB). }
$$

En el CB no existe accountability, la Universidad de Concepción se apropió del centro y trabaja bajo sus propios fines, en cambio la UDT al tener asociado fondos basales provenientes del Estado, está obligada a rendir cuentas.

\section{Recursos claves}

En general, ambos centros cuentan con investigadores y profesionales altamente calificados, siendo el recurso principal de cada uno de los centros. Pero los enfoques y condiciones en que se desarrolla el personal difieren entre ambos centros. Por un lado, los investigadores que trabajan en el CB se encuentran adscritos a la Facultad de Ciencias Forestales o 
a la Facultad de Ciencias Naturales y Oceanográficas, por lo que deben distribuir sus funciones investigativas con su labor docente, difuminando los bordes entre las responsabilidades con las facultades y las responsabilidades con el centro. Esta situación no ocurre en la UDT, donde los investigadores están dedicado en un $100 \%$ a la investigación y gestión de sus proyectos, focalizándose completamente en ellos.

Por otro lado, la infraestructura de cada uno de los centros cumple funciones diferentes. Por una parte, el CB cuenta con laboratorios con equipamiento adecuado para el desarrollo de investigación y procesamientos analíticos complejos. La UDT, por su parte, tiene capacidades de escalamiento y pilotaje, aspecto necesario y atractivo tanto para los emprendedores como para las empresas, que le permiten ser un foco de atención de la industria, situación que se presenta en menor medida en el CB. Es por ello que entre ambos centros existe una alianza estratégica para potenciar su labor, ofreciendo el CB sus capacidades analíticas y la UDT sus capacidades de escalamiento y pilotaje.

También se aprecian diferencias en los enfoques, uno enfocado en la investigación aplicada y el otro enfocado a la investigación básica. Al analizar los equipos de trabajo con que cada centro cuenta, la UDT desde la adjudicación del proyecto basal, formó un área de transferencia tecnológica compuesta por un ingeniero encargado de área y tres ingenieros de transferencia tecnológica, que han resultado un recurso fundamental para el mejoramiento de los indicadores de transferencia del quehacer del centro. Por otra parte, el CB, hasta el año pasado, contaba con solo un ingeniero de gestión, encargado de todas las labores de transferencia tecnológica, situación que se ha buscado mejorar mediante un proyecto adjudicado el año 2014.

Otro recurso que ha sido determinante en las diferentes performances que ha tenido cada uno de los centros, son las características del liderazgo directivo. Por un lado, la UDT es dirigida por un perfil más gerencial, con habilidades motivadoras, con larga experiencia en la generación de vínculos y trabajos con la industria. Por el otro, el CB cuenta con un director más bien democrático, lo que fomenta el descubrimiento y desarrollo de nuevas áreas de investigación, pero a su vez difumina la misión y resultados del centro.

\section{Actividades claves}

Si bien la UDT y el CB tienen objetivos similares, la forma de llevarlos a cabo varía mucho entre un centro y otro. Por un lado, el CB ha enfoca- do sus actividades en el desarrollo de investigación básica y aplicada, donde la producción de artículos científicos es un resultado característico de esta labor. La UDT, por su parte, se ha enfocado en el desarrollo tecnológico, siendo el patentamiento y transferencia tecnológica reflejo de ello. El no estar inserto dentro de la Universidad ha obligado a la UDT a desarrollar capacidades internas de transferencia tecnológica, generando instancias de trato directo con las empresas para que esta ocurra y no como lo realiza el CB por medio de la oficina de transferencia tecnología de la Universidad de Concepción.

Otra actividad que diferencia a un centro del otro es la etapa inicial de concepción de idea. Por un lado, la UDT ocupa gran parte de su tiempo en levantar información sobre las necesidades de las empresas, se relaciona directamente con ellas y se anticipa al mercado, mostrando una actitud más proactiva. En cambio, en el CB un proyecto de investigación nace del interés particular de un investigador, quien debe cerciorarse de la viabilidad comercial de la investigación, pero quien no necesariamente realiza un trabajo previo para identificar las reales necesidades de la empresa. Además, al desarrollar proyectos con empresas más grandes con equipos de investigación internos, no existe un proceso de transferencia tecnológica formal, ya que los avances en la investigación y desarrollo de nuevos productos y procesos son absorbidos por la empresa durante el proceso, prescindiendo de una etapa formal de transferencia tecnológica.

La adjudicación de proyectos es otra actividad que diferencia a ambos centros, si bien ambos tienen una buena tasa de adjudicación, los instrumentos utilizados difieren de las capacidades y actividades necesarias para su adjudicación. La UDT se ha caracterizado por adjudicarse, en su mayoría, fondos provenientes de Corfo, quienes tienen como fin financiar proyectos que impacten directamente a la industria, ya sea mediante ciencia aplicada, desarrollo de nuevas líneas de producto, transferencia tecnológica, emprendimientos, entre otros. De esta forma, sus capacidades y actividades tienen un fuerte enfoque comercial y aplicado, siendo la vinculación y levantamiento de las necesidades de la industria, como las actividades de pilotaje y escalamiento de emprendimientos, aspectos fundamentales para lograr adjudicarse los fondos necesarios. En cambio, el CB se ha caracterizado por adjudicarse, en su mayoría, fondos prevenientes de Conicyt, quienes financian gran parte de la investigación en ciencias básicas y aplicadas que se desarrolla en el país. Esto significa para el $\mathrm{CB}$ un fuerte enfoque académico en las capacidades y actividades realizadas por el centro, en perjuicio de su vinculación e impacto en el sector productivo. 
Aliados claves

Dada la estructura de financiamiento de ambos centros, uno de sus principales aliados claves son las agencias del Estado encargadas de promover el desarrollo de la ciencia y tecnología, y fomentar el desarrollo productivo. Alrededor del $70 \%$ de los proyectos desarrollados por los centros, es financiado con fondos públicos, por lo que alinearse con los objetivos de las agencias, conocer sus estructuras e intereses y construir vínculos de apoyo con las entidades locales, como el gobierno regional, es fundamental para aumentar la tasa de efectividad de adjudicación de proyectos y así poder seguir funcionando.

Otro aliado clave es la Universidad de Concepción, pero con un rol muy diferente para cada centro. Para el CB, la Universidad de Concepción ampara al centro financiera e institucionalmente, provee recursos para costear la operación del centro y permite a los académicos de algunas de sus facultades realizar media jornada laboral en el centro, además de brindar el prestigio que caracteriza a la Universidad de Concepción a nivel regional y nacional. En cambio, la UDT desde un inicio se ha desarrollado autónomamente, si bien mantiene el vínculo con la Universidad de Concepción, en los hechos ese vínculo favorece más a la Universidad que al centro, ya que la universidad no apoya financieramente al centro, siendo este último quien debe pagar un "impuesto" a la Universidad. Al igual que el CB, se vincula con académicos de la Universidad, principalmente de la facultad de ingeniería, sin embargo estos no disponen de horas asignadas por la Universidad para realizar trabajos en la UDT.

\footnotetext{
"Uno de los factores que ha favorecido el trabajo de la UDT es que la Universidad sea un agente facilitador y no obstructor."
}

(A.B., Directivo, UDT).

Finalmente, si bien se ha intentado fomentar las alianzas internacionales, el impacto que estas iniciativas han tenido no es de gran relevancia, siendo solo destacable los vínculos generados por la UDT con los institutos de la organización alemana Fraunhofer Gesellschaft, quienes además de servir como inspiración de modelo de negocio del centro, ha fomentado el desarrollo de proyectos conjuntos y el flujo de académicos y estudiantes de postgrado.

\section{Estructura de costos}

La estructura de costos es similar en ambos centros, gran parte de los costos están representados por las remuneraciones de los recursos humanos. La UDT tiene una planta de investigadores, profesionales y técnicos que llega a 120 personas; a quienes debe pagar sueldos, gastos sociales y bonificaciones, representando cerca del $60 \%$ de los costos totales del centro. En cambio, el CB tiene una planta de trabajadores menor, de los cuales una parte importante de ellos mantiene contrato directamente con la Universidad de Concepción, debiendo el centro costear una asignación respectiva. Los gastos en recursos humanos representan cerca del $50 \%$ de los costos totales del centro.

Los costos de los proyectos están determinados por la convocatoria respectiva, por lo que su importancia en la estructura de costo es variable.

Finalmente, algunos indicadores que resumen los resultados de ambos centros de investigación se presentan en las siguientes figuras:

\section{Conclusiones}

En relación a los modelos de negocio analizados, se puede concluir que las principales diferencias entre ambos centros de investigación, que explican en parte las diferencias en los resultados e impacto al desarrollo económico de la región, se relacionan con 4 elementos del Canvas: Segmento de clientes/usuarios, Propuesta de valor, Recursos claves y Actividades claves.

Respecto al segmento de clientes/usuarios, la UDT se ha vinculado con empresas medianas y pequeñas de la industria relacionada con la biomasa forestal, generando confianzas con aquellas que carecen de las capacidades para mejorar sus procesos y desarrollar nuevos productos por sí solos. Son este tipo de empresa las que representan mayor número, emplean a la mayor cantidad de personas y presentan menores niveles de desarrollo. Por esta razón, las probabilidades de impactar en el desarrollo económico local a través de estas empresas, son mayores que relacionándose con empresas más grandes. Por otro lado, están los emprendedores. Este segmento de clientes/usuarios es uno de los principales focos de atención de la política pública, por lo que la UDT, a diferencia del CB, le ha dado un lugar prioritario dentro de su cartera de clientes. La mejora y desarrollo de nuevas ideas proveniente de emprendedores, aumenta las posibilidades de diversificar la matriz productiva de la región. 
Figura 1: $\mathrm{N}^{\circ}$ de solicitudes de patentes

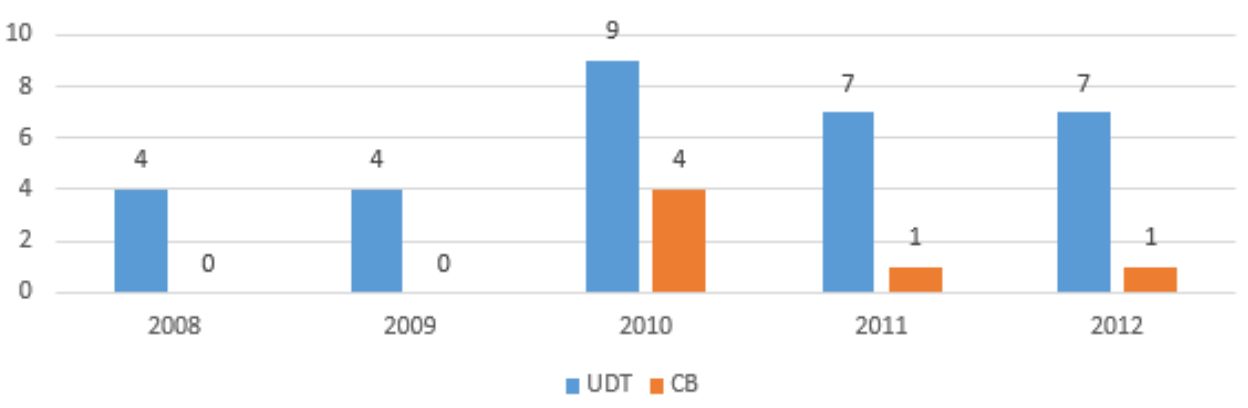

Fuente: Elaboración propia

Figura 2: $\mathrm{N}^{\circ}$ de publicaciones

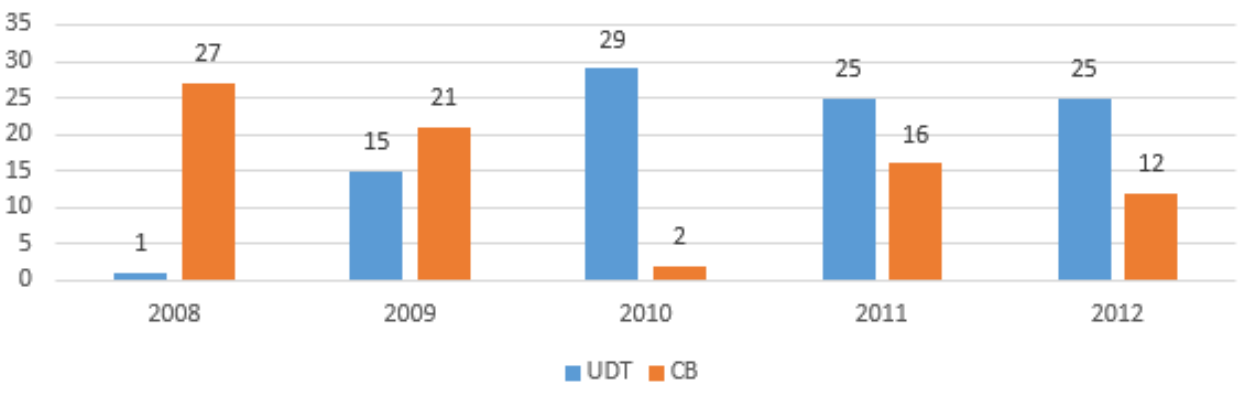

Fuente: Elaboración propia

Figura 3: $\mathrm{N}^{\circ}$ de proyectos ejecutados

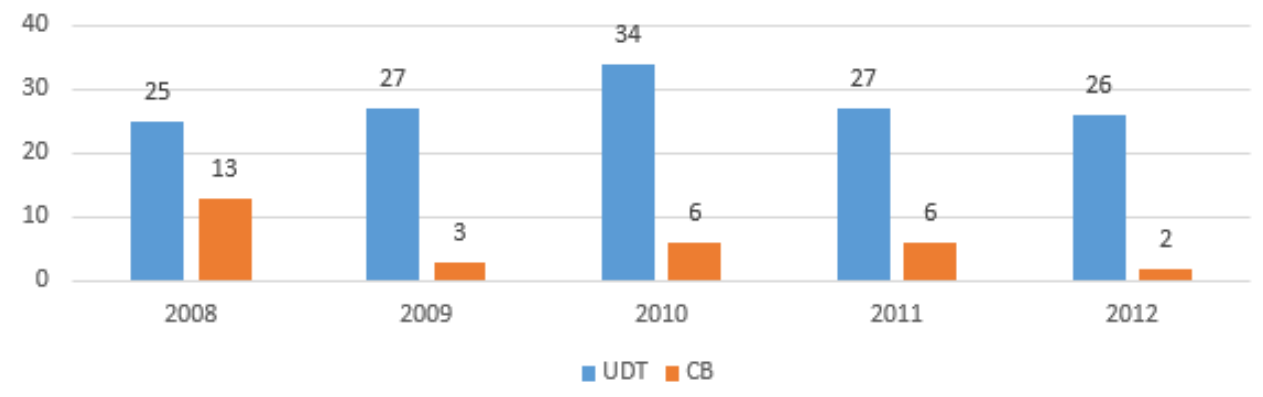

Fuente: Elaboración propia 
Respecto a las propuestas de valor, la UDT ha aprendido a ofrecer al sector productivo soluciones acordes a la realidad del contexto productivo local, en cuanto de recursos, tiempos de desarrollo, aplicabilidad y procesos administrativos, por mencionar algunos. A diferencia del CB, que se ha enfocado en ofrecer a las grandes empresas proyectos de investigación y desarrollo de gran envergadura y a largo plazo, desde una perspectiva más bien académica y con necesidades de inversiones más altas. Esta propuesta de valor ha interesado a más de alguna gran empresa, la cual ha debido asumir los tiempos, costos y burocracia propia de la Universidad.

Respecto a los recursos claves, se pueden mencionar tres componentes que hacen la diferencia entre un centro y otro: Las características de los recursos humanos, el enfoque de la infraestructura y equipamiento disponible y el estilo de liderazgo.

- Los recursos humanos de la UDT son en su mayoría, salvo los jefes de área y el director, profesionales e investigadores jóvenes, quienes ven al centro como una gran escuela para el propio desarrollo profesional. Su dedicación es jornada completa en el centro, con un enfoque permanente a la empresa y sus necesidades. El recurso humano de $\mathrm{CB}$, en cambio, son investigadores pertenecen a alguna facultad de la universidad, por lo que deben compartir sus labores de investigación en el centro, con sus labores de docencia y gestión en su respectiva facultad. Son profesionales con vasta trayectoria académica que acostumbran a desarrollar su trabajo según sus propios intereses.

- El equipamiento e infraestructura de ambos centros también es un elemento diferenciador cuando lo relacionamos con el impacto en el sector productivo. El equipamiento e infraestructura de la UDT está enfocado en el desarrollo de procesos y productos, con parámetros cercanos a los presentados por la industria, lo que invita a diferentes empresas a trabajar con la UDT y utilizar la infraestructura y equipamiento disponible, así como a invertir en nuevo equipamiento. Mientras que el CB está dotado de una serie de laboratorios de primer nivel, lo que permite realizar una serie de experimentos y análisis complejos; sin embargo, esta complejidad se presenta principalmente en empresas grandes con procesos y productos más avanzados, por lo que son estas empresas las que finalmente reciben al $\mathrm{CB}$ como aliado. Cabe mencionar que el emplazamiento de cada uno de estos centros también determina la vinculación con el sector productivo, por un lado está la UDT ubicada en el parque industrial de Coronel, compartiendo ubicación geográfica con grandes, medianas y pequeñas empresas relacionadas con la biomasa forestal; mientas que por otro lado está el CB, emplazado en el Campus central de la Universidad de Concepción, con la lejanía física y simbólica que esto significa para el sector industrial.

- Los estilos de liderazgos también diferencian los resultados de ambos centros. La dirección de la UDT se caracteriza por tener un estilo gerencial, constructor de redes y levantador de recursos, fomenta el trabajo en equipo y mantiene a su equipo de trabajo constantemente motivado, interesado y al tanto de los trabajos que se están realizando por el centro en su conjunto, mientras que la dirección del CB es más bien horizontal, cumpliendo el director un rol más bien administrativo que gerencial, al estar compuesto el equipo de trabajo en su mayoría por académicos, existe mayor autonomía para el desarrollo de líneas de investigación personales, diversificando, pero a su vez difuminando el foco de investigación del centro.

Finalmente, respecto a las actividades claves de cada uno de los centros, estas se diferencian desde la concepción de la idea de investigación, hasta el fin que buscan con el desarrollo de ésta, significando una diferencia sustancial en los resultados conseguidos por ambos centros, en relación al impacto y vinculación con el sector productivo de la región. Por un lado, la UDT antes de comenzar cualquier proyecto de investigación levanta toda la información necesaria sobre las necesidades actuales y futuras de la industria con la que trabaja, identificando posibles soluciones a problemas reales. Luego, desarrolla su proyecto en conjunto con la industria, cuyo fin último es la transferencia de un desarrollo tecnológico que mejore la productividad particular de la empresa. Por otra parte, en el CB las ideas nacen del interés personal de los investigadores que trabajan en el centro, respondiendo a una línea de investigación desarrollada durante su trayectoria académica, esta idea se le ofrece a distintas empresas, identificando interesados en levantar un proyecto en conjunto. Dado la dependencia laboral de los investigadores con la Universidad de Concepción, uno de los fines principales del desarrollo de estos proyectos es la producción científica, traducida en publicaciones en revistas indexadas, pues el desempeño de los académicos en la Universidad de Concepción está altamente vinculado a su capacidad de producir artículos, independiente de su vinculación e impacto en el medio productivo, social y cultural en el que se insertan.

En relación al rol de estos dos centros de investigación como componentes de la esfera academia 
del modelo de la triple hélice, ambos se encuentran claramente delimitados y especializados en sus funciones, cumpliendo principalmente los dos roles históricos de la universidad,: la formación de profesionales y la investigación. Ambos presentan un nivel de madurez alto con grandes capacidades instaladas para la formación de capital humano avanzado, desarrollo de investigación de primer nivel e incluso la posibilidad de vincularse activamente e impactar en el desarrollo del medio productivo de la región. Sin embargo, la vinculación con el medio externo, tercera misión de las universidades, aún no se ha instalado en la cultura de estos dos centros, pese a existir los instrumentos y una oficina especializada enfocada en vincularlos con el sector productivo.

\section{Recomendaciones}

En base a lo desarrollado durante el artículo, se realizan dos recomendaciones de cara a mejorar el desempeño de la política pública en cuanto al desarrollo local y al rol que puede cumplir la Universidad en este proceso.

En primer lugar, se recomienda fomentar la generación de confianzas y asociatividad entre los diferentes actores, y pasar de un espacio de conocimiento a un espacio de consenso, donde los diferentes actores propongan, discutan y evalúen diferentes proyectos de investigación y desarrollo que vayan en beneficio de la sociedad y no solo en un beneficio particular. Es labor de la política pública, generar los mecanismos para dinamizar la matriz productiva, disminuyendo las barreras de entrada de empresas productivas y la salida de empresas no productivas.

En segundo lugar, se recomienda, dadas las características y posibilidades que tiene la región y las capacidades de la Universidad de Concepción, la creación de un centro de extensión tecnológica enfocada en fortalecer a las PYME's relacionadas con los sectores productivos que tienen menor volúmenes de ventas por trabajador dependiente, optimizando sus procesos de producción, mejorando las asimetrías de información y solucionando los problemas de coordinación, reduciendo así los costos individuales de mejora. Por otro lado, se debe buscar diversificar la matriz productiva de la región, potenciando la incubadora que actualmente tiene la universidad, que se ha enfocado en emprendimientos, principalmente de base tecnológica.

\section{Referencias}

Banco Central de Chile (2013). Cuentas Nacionales de Chile. Banco Central de Chile.

De Gregorio, J. (2007). Macroeconomía: Teoría y políticas. Pearson.

Etzkowitz, H. y Leydesdorff, L. (2000a). The dynamics of innovation: from national systems and "mode 2" to a triple helix of universityindustry-government relations. Research policy, 29(2):109-123.

Etzkowitz, H., Webster, A., Gebhardt, C., y Terra, B. R. C. (2000b). The future of the university and the university of the future: evolution of ivory tower to entrepreneurial paradigm. Research policy, 29(2):313-330.

Osterwalder, A. y Pigneur, Y. (2011). Generación de modelos de negocio: un manual para visionarios, revolucionarios y retadores. Planeta.

Palacios, O. (2005). Los evolucionistas o neoschumpeterianos. Mundo siglo XXI, (1):87-113. 